indicating a relative increase in the risk of death of about $17.2 \%$.

The authors conclude that kidney function level can be used to predict mortality among patients with CVD who have undergone cardiac catheterization, and state that kidney function should, therefore, be assessed in all CVD patients.

Original article Hemmelgarn BR et al. (2006) Refined characterization of the association between kidney function and mortality in patients undergoing cardiac catheterization. Eur Heart J 27: 1191-1197

\section{Warfarin is not appropriate in all patients with AF-associated ischemic stroke}

Warfarin considerably reduces the risk of recurrent stroke in patients who have had an ischemic stroke and are in atrial fibrillation (AF). Researchers in New Zealand have investigated the reasons why, despite the proven benefits, only about $50 \%$ of patients with AF are started on warfarin therapy following ischemic stroke.

The analysis included 93 patients (mean age 80 years) admitted to Auckland City Hospital with ischemic stroke and either permanent or paroxysmal AF. The diagnosis of AF was known in 64 (69\%) patients before admission; the other patients were diagnosed during admission. Of the 64 patients in the former group, just 13 (20\%) were taking warfarin. In total, 17 patients died in hospital. At discharge, warfarin was recommended in 35 of the 76 surviving patients: 28 were already taking warfarin, 4 started warfarin after discharge and 3 did not commence treatment. Compared with those taking warfarin, patients who did not take warfarin were more likely to be dependent on others or to require institutional care at discharge. The most common reasons for not starting warfarin at discharge were severe disability and frailty, high risk of falls and short life expectancy.

The authors conclude that, in their study, underutilization of warfarin mostly occurred before stroke onset; by the time stroke had occurred, warfarin administration was precluded in approximately $50 \%$ of patients. They state that decisions regarding warfarin therapy in stroke patients with AF should be made on the basis of individual assessment.

Original article Somerfield J et al. (2006) Not all patients with atrial fibrillation-associated ischemic stroke can be started on anticoagulant therapy. Stroke 37: 1217-1220

\section{Lipid-lowering PCSK9 sequence variants protect against coronary heart disease}

Although genetic studies might help to clarify whether low LDL cholesterol levels confer protection against coronary heart disease (CHD), disorders in which LDL cholesterol levels are substantially lowered seem to be genetically heterogeneous.

Recently, Cohen et al. identified two sequence variations in the proprotein convertase subtilisin/kexin type 9 (PCSK9) gene among black individuals and another mutation in the PCSK9 gene in white people. They noted that these variations were associated with reduced plasma levels of LDL cholesterol. This research group now report their findings from a 15-year follow-up study, undertaken to assess the effect of these sequence variants on LDL cholesterol levels and the risk of $\mathrm{CHD}$ in a cohort of participants from the Atherosclerosis Risk in Communities (ARIC) study.

The investigators recorded the annual incidence of CHD (myocardial infarction, fatal $\mathrm{CHD}$, or coronary revascularization) and subclinical atherosclerosis, and then screened the PCSK9 gene for mutations. Nonsense mutations were found in $2.6 \%$ of black participants $(n=3,363)$, with associated reductions in LDL cholesterol $(28 \% ; P=0.001)$ and risk of CHD $(88 \% ; P=0.008)$ compared with noncarriers of the mutation. A mutation in PCSK9 was identified in $3.2 \%$ of white individuals $(n=9,524)$, which was linked with a $15 \%$ reduction in LDL cholesterol and a halved risk of $\mathrm{CHD}(P=0.003)$ compared with noncarriers.

It is unknown whether the beneficial effects of low LDL cholesterol result in an overall reduction in mortality rates, but these promising data suggest that relatively moderate reductions in LDL cholesterol can markedly reduce CHD in the population.

Original article Cohen JC et al. (2006) Sequence variations in PCSK9, low LDL, and protection against coronary heart disease. N Engl J Med 354: 1264-1272

\section{Detection of high-risk atherosclerotic plaques by palpography}

Acute coronary events frequently result from atherosclerotic plaque rupture at apparently 